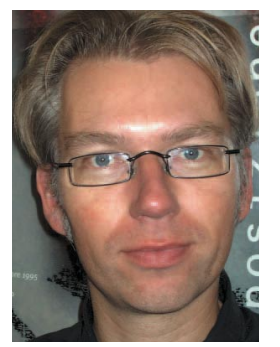

Hans Jörgen Grabe

\title{
Körperliche Symptomatik ohne Ursache - Somatisierungsstörung?
}

\author{
Hans Jörgen Grabe und Harald Jürgen Freyberger
}

Klinik und Poliklinik für Psychiatrie und Psychotherapie der Ernst-Moritz-Arndt-Universität Greifswald im Klinikum der Hansestadt Stralsund

psychoneuro $2003 ; 29$ (11): 513-517

S omatoforme Störungen sind durch körperliche Beschwerden und Symptome gekennzeichnet, für die keine oder keine ausreichenden organischen Befunde als Erklärung erhoben werden können. Diese Beschwerden werden wiederholt dargeboten und gehen meist mit hartnäckigen Forderungen nach weiteren medizinischen Untersuchungen trotz wiederholter negativer Ergebnisse einher. Ärztliche Erklärungen über das Fehlen organischer Ursachen oder die Möglichkeit einer psychischen Genese werden vom Patienten zumeist nicht akzeptiert (1). Typische Störungsbilder dieser Kategorie stellen die (undifferenzierte) Somatisierungsstörung, die somatoforme Schmerzstörung, die funktionellen autonomen Funktionsstörungen, die Hypochondrie und dissoziative Störungen dar (3).

Als auslösende Faktoren werden vor allem externe Belastungsfaktoren (z.B. Beruf, Familie), konflikthafte Entwicklungsprozesse, psychovegetative Labilität und Störungen kognitiver und emotionaler Wahrnehmungsprozesse im Sinne des Alexithymiekonzepts diskutiert. Gerade bei der Diagnosengruppe der somatoformen Störungen hat es in den letzten Jahren neue diagnostische Entwicklungen gegeben, die zum einen der Vielfalt dieser

Somatoforme Störungen weisen mit Lebenszeit-Prävalenzraten bis zu 20\% und vielfach chronischen Verläufen eine weite Verbreitung in der ärztlichen Praxis auf. Die Fixierung auf die körperliche Beschwerdesymptomatik, für die sich keine oder keine ausreichende somatische Ursache finden lässt, bringt Ärzte und Betroffene in eine schwierige Situation: Oft endet der unbefriedigende Kontakt, nachdem eine ganze Kaskade diagnostischer Maßnahmen abgearbeitet wurde, mit dem Wechsel zu einem Spezialisten, der „die richtige somatische Diagnose schon herausfinden wird“. Es werden neben neuen epidemiologischen Daten handlungspraktische Kriterien und Strategien zur Diagnosenstellung, zur Primärintervention und zum Umgang und mit betroffenen Patienten vorgestellt. Letztendlich dienen die rasche richtige diagnostische Einschätzung und die sich daraus ableitenden Therapiemöglichkeiten der Heilung dieser Patienten und der Verhinderung der häufigen Chronifizierung.

Störungssymptome aber auch ihrer epidemiologischen und gesundheitsökonomischen Bedeutung gerecht zu werden suchen.

Die Betrachtung epidemiologischer Daten zur Prävalenz somatoformer Störungen lässt die Bedeutung und Häufigkeit dieser Störungen in der allgemeinärztlichen Praxis erahnen.

\section{Aktuelle epidemiologische Ergebnisse}

In einer in Lübeck und Umgebung durchgeführten Studie zur Häufigkeit von psychischen Krankheitssymptomen und Diagnosen nach DSM-IV an 4075 Probanden zeigte sich eine Lebenszeitprävalenz von $19,6 \%$ für die undifferenzierte Somatisierungsstörung $(7,8)$. Zusätzlich litten $12,3 \%$ während ihres bisherigen Lebens unter einer schweren somatoformen Schmerzstörung (mit erheblichem Leidensdruck oder Beeinträchtigung). Die 6Monats-Prävalenz lag bei $5,4 \%$. Weniger stringente diagnostische Kriterien (DSM-III-R) ergaben sogar Lebenszeitprävalenzen der somatoformen Schmerzstörung bis zu 33,7\%. 95\% aller Betroffenen gaben an, wegen der Schmerzsymptome einen Arzt (i.d.R. Hausarzt und Allgemeinmediziner) kontaktiert zu haben (7).

In einer anderen Studie gingen wir der Hypothese nach, dass diese Patientengruppe vermutlich ein deutlich höheres Inanspruchnahmeverhalten ärztlicher Behandlungen an den Tag legt, wodurch der effektive ärztliche Kontakt mit diesen Patienten überproportional häufig stattfindet. Die in Stralsund und Greifswald durchgeführte SHIP-Studie (Study of Health in Pomerania) 
mit 4310 erfassten Probanden aus der Allgemeinbevölkerung zeigte eine Prävalenz somatoformer Syndrome von 16,1\%. 18,2\% der Patienten in allgemeinärztlichen Praxen litten u.a. unter einem somatoformen Syndrom, in internistischen waren es $25,1 \%$, in chirurgischen $21,3 \%$ und in orthopädischen 27,6\%. Die tatsächlichen Konsultationshäufigkeit lag aber über dem Durchschnitt der anderen Patienten: So beanspruchten Patienten mit einem somatoformen Syndrom $32,5 \%$ aller Termine in Allgemeinarztpraxen, $41 \%$ in internistischen Praxen, 49,5\% in chirurgischen Pra- xen und $51,7 \%$ in orthopädischen Praxen.

Ältere Studien schätzten den Anteil der Patienten mit psychosomatischen Störungen in einer haus- oder allgemeinärztlichen Praxis auf etwa $10 \%$ ein $(2,11)$. Diese Diskrepanz lässt sich nur zum Teil mit methodischen Unterschieden der Studien erklären, eine Zunahme dieser Störungsgruppe im Gesundheitsystem ist begründet anzunehmen. Die erhebliche gesundheitsökonomische Bedeutung dieser Daten ist offensichtlich, ein professionellerer Umgang mit diesen Störungen ist zwingend.

\section{Tab. 1 Somatische Beschwerdesymptomatik}

Symptomatik

aus neurologisch-
psychiatrischen
Praxen
$(\mathrm{N}=1183)$

\begin{tabular}{l}
\hline Kopfschmerzen \\
\hline Herzklopfen \\
\hline Sodbrennen, Aufstoßen, Magendrücken \\
\hline Starke Blähungen, Flatulenz \\
\hline
\end{tabular}

Rückenschmerzen

Schwindel

Müdigkeit und Leeregefühl im Kopf

Mundtrockenheit

Andauernde Müdigkeit

Gliederschmerzen

Schlafstörungen

Taubheit, Kribbeln

Atemnot

\begin{tabular}{|l}
787 \\
837 \\
\hline 480 \\
\hline 223 \\
\hline 615 \\
\hline 817 \\
767 \\
\hline 182 \\
\hline 455 \\
\hline 514 \\
\hline 984 \\
\hline 400 \\
\hline 357 \\
\hline
\end{tabular}

\begin{tabular}{|l|}
\hline $67 \%$ \\
\hline $71 \%$ \\
\hline $41 \%$ \\
\hline $19 \%$ \\
\hline $52 \%$ \\
\hline $69 \%$ \\
\hline $65 \%$ \\
\hline $15 \%$ \\
\hline $39 \%$ \\
\hline $43 \%$ \\
\hline $83 \%$ \\
\hline $34 \%$ \\
\hline $30 \%$ \\
\hline
\end{tabular}

823
927
654
479
700
854
890
274
634
466
1104
448
346

Anzahl der Patienten aus internistischallgemeinmedizinischen Praxen $(\mathrm{N}=1324)$ 823 927 654 479 700 854 890 274 634 466 1104 448 346

\begin{tabular}{|l}
\hline $62 \%$ \\
\hline $70 \%$ \\
\hline $49 \%$ \\
\hline $36 \%$ \\
\hline $53 \%$ \\
\hline $65 \%$ \\
\hline $67 \%$ \\
\hline $21 \%$ \\
\hline $48 \%$ \\
\hline $35 \%$ \\
\hline $83 \%$ \\
\hline $34 \%$ \\
\hline $26 \%$ \\
\hline
\end{tabular}

Somatische Beschwerdesymptomatik der letzten 6 Monate bei Patienten mit somatoformen Störungen in neurologisch-psychiatrischen und allgemeinmedizinisch-internistischen Praxen $(4,5)$

\section{Tab. 2 Relevante Belastungsfaktoren}

\begin{tabular}{lrrrr} 
Belastungsfaktoren & $\begin{array}{c}\text { Patienten aus neurologisch- } \\
\text { psychiatrischen Praxen } \\
(\mathrm{N}=964)\end{array}$ & $\begin{array}{c}\text { Patienten aus } \\
\text { internistisch-allgemein- } \\
\text { medizinischen Praxen } \\
(\mathrm{N}=1075)\end{array}$ \\
\hline Beruf/Schule & 341 & $35 \%$ & 328 & $31 \%$ \\
\hline Finanzielle Probleme & 175 & $18 \%$ & 217 & $20 \%$ \\
\hline Familiensituation & 461 & $48 \%$ & 599 & $56 \%$ \\
\hline Problematische Kindheit & 162 & $17 \%$ & 126 & $12 \%$ \\
\hline Gegenwärtige Partnerschaft & 308 & $32 \%$ & 339 & $32 \%$ \\
\hline Frühere Partnerschaft & 86 & $9 \%$ & 89 & $8 \%$ \\
Sonstiges & 133 & $14 \%$ & 100 & $9 \%$ \\
\hline
\end{tabular}

Relevante Belastungsfaktoren bei Patienten mit somatoformen Störungen in neurologischpsychiatrischen und allgemeinmedizinisch-internistischen Praxen $(4,5)$

\section{Diagnostik der somato- formen Störungen in der Allgemeinarztpraxis}

Neben dem ärztlichen diagnostischen Gespräch bieten sich einfache Fragebogenverfahren zum Selbstausfüllen zur orientierenden Diagnostik an (z.B. Screening für somatoforme Störungen (SOMS) von Rief, Hiller und Heuser oder als umfassenderes Screening-Instrument für Psychopathologie die Symptom CheckListe (SCL-90-R) von Franke). Diese kann der Patient im Wartezimmer vor der Konsultation eigenständig ausfüllen. Grundlage der diagnostischen Validität dieser Verfahren stellen die zumeist multiplen körperlichen Beschwerden dar, die in den angegebenen Kombinationen verschiedener betroffener Organsysteme oft schon auf den ersten Blick nur höchst unwahrscheinlich einer klar definierten körperlichen Erkrankung entsprechen (Tab. 1). Die auffallend hohe Assoziation mehrerer somatischer Symptome bei der Mehrzahl der Patienten mit somatoformen Beschwerden konnte von uns in einer naturalistischen Therapiestudie in neurologisch-psychiatrischen (5) und internistischallgemeinmedizinischen Praxen (4) bestätigt werden: 90\% der Patienten beklagten mehr als drei Symptome, 45-50\% mehr als fünf Symptome. Bei 95\% der Patienten hielten die Symptome einen Monat oder länger an. Diagnostisch wegweisend ist also die Erfassung möglichst vieler Symptombereiche. Berichtet der Patient aufgrund eines nicht unüblichen Zeitmangels nur einen Teil seiner tatsächlichen Beschwerdesymptomatik, so kann dies viel eher zu rein somatischen Arbeitsdiagnosen führen. Dies wiederum kommt einem abrechnungstechnisch günstigen und zeitökonomisch effektiven strukturierten weiteren somatodiagnostischen und somato-therapeutischen Vorgehen entgegen, wird aber dem betroffenen Patienten nicht wirklich helfen.

Weiterhin wegweised können zusätzliche psychische Beschwerdesymptome sein: Unsere Daten zeigen hohe Komorbiditätraten auf Syndromebene $(4,5)$ : Bei über $90 \%$ der untersuchten Patienten wurden 
psychische Symptome festgestellt. Rund drei Viertel der Patienten litten unter Unruhe und Spannungsgefühlen sowie unter Schlafstörungen, etwa zwei Drittel unter Angstsymptomen. Mehr als jeder Zweite litt unter Depressivität, rund jeder Dritte unter Konzentrationsstörungen, etwa jeder Vierte unter Reizbarkeit und Aggressivität und zirka jeder Fünfte unter Dysphorie.

Zusätzlich finden sich sehr häufig aktuelle familiäre oder berufliche Konfliktsituationen (Tab. 2). Diese können in einem vertraulichen Gespräch auch und gerade von Hausoder Allgemeinärzten erhoben und benannt werden. Das Vorliegen einer psychosozialen Belastungs- oder Konfliktsituation liefert beim Fehlen eindeutiger somatischer Krankheitsursachen ebenfalls einen wichtigen diagnostischen Hinweis für das Vorliegen einer somatoformen Störung.

I

Therapiestrategien der somatoformen Störungen in der Allgemeinarztpraxis Frühzeitige Diagnosestellung!

Somatoforme Störungen zeigen eine erhebliche Tendenz zur Chronifizierung. Neben den primären Mechanismen, die für den „Somatisierungsprozess" verantwortlich sind, kommt der iatrogenen Verstärkung und Fixierung der Symptomatik durch ein somatisches Krankheitsverständnis der Ärzte eine erhebliche Bedeutung zu, z.B. durch entsprechende z.T. aufwändige apparative Untersuchungen die fast garantiert irgendwelche Diagnosen liefern, die sich im Krankheitsverständnis der Patienten fest etablieren. Nach körperlicher Untersuchung, ausreichender Klärung der Vorbefunde und Anameseerhebung sollte der tätige Arzt nicht zögern, dem Patienten seine Verdachtdiagnose mitzuteilen. Aber niemals so: „Sie haben nichts!“

\section{Vertrauensbildende psychosoziale Anamnese}

Diese kann entscheidende Hinweise auf die Genese der Störung liefern und dient dazu, dass der Patient sich in seiner Lebenssituation gehört und beachtet fühlt. Der Patient erkennt, dass er nicht nur für den Arzt aufgrund seiner komplexen Beschwerdesymptomatik und der schwierigen differenzialdiagnostischen Situation interessant ist! Allerdings werden viele Patienten von sich aus nicht ohne weiteres objektive Stressoren als subjektive Stressoren wahrnehmen und akzeptieren.

\section{Angemessene somatische Abklärung}

Um den Patienten in seinen Ängsten ernst zu nehmen und ggf. wichtige somatische Diagnosen auszuschließen, sollte eine angemessene Diagnostik erfolgen. Allerdings sollte, wenn möglich schon vorher, der Verdacht geäußert werden, dass z.B. „die erhebliche Stressbelastung in der letzten Zeit einige Körperfunktionen und die Körperwahrnehmung durcheinander gebracht haben“.

\section{Einfaches psychosomatisches} Krankheitskonzept vermitteln

Falls nach den angemessenen Untersuchungen keine wirklich konklusiven Befunde erhoben wurden und die gesamte übrige Konstellation (komplexe Beschwerdesymptomatik, psychosoziale Belastung, psychiatrische Komorbidität) für ein psychosomatisches Krankheitsgeschehen spricht, sollte auf weitere somatische Untersuchungen konsequent verzichtet werden und der Fokus auf die Erarbeitung eines einfachen Krankheitskonzepts und weiterer Therapieoptionen gelegt werden. Einfache Modelle des eigentlich sehr komplexen und noch in weiten Teilen unverstandenen Mechanismus der Somatisierung wären z.B. „Stress hat die Regulation von Körperfunktionen durch das Nervensystem durcheinander gebracht" oder „die Seele drückt ihre Not durch Schmerzen aus“ oder „Ihre Trauer über (...) zeigt sich immer noch als dieses oder jenes Symptom“.

\section{Prinzipielle Therapieoptionen diskutieren}

Die im Folgenden skizzierten Therapieoptionen sollten mit dem Patienten frühzeitig erörtert werden. Zum einen entwickelt der Patient selbst oft einen speziellen Therapiewunsch, zum anderen sollte bei unbefriedigenden Ergebnissen des ersten Therapieversuchs auch schon weitere Möglichkeiten einschließlich der Behandlung durch den Facharzt oder Psychotherapeuten angesprochen werden.

\section{Medikamentöse Therapie}

Bei einem zusätzlichen Bestehen von psychischen Auffälligkeiten wie Angst und Depression bietet sich eine psychopharmakologische Therapie mit Antidepressiva (z.B. selektive Serotonin-Wiederaufnahmehemmer wie Citalopram, Sertralin oder Paroxetin) an. Weiterhin hat sich Opipramol (Insidon ${ }^{\circledR}$ ) in einer plazebo-kontrollierten Studie (10) bei Patienten mit generalisierter Angststörung und Patienten mit somatoformer Störung als signifikant wirksam erwiesen. Eine direkte günstige Wirkung auf somatoforme Beschwerden konnte auch in zwei umfangreichen Anwendungsbeobachtungen unter naturalistischen Praxisbedingungen nachgewiesen werden $(4,5)$. Auf jeden Fall sollte auf eine Gabe von Benzodiazepinen verzichtet werden. Diese wirken nicht kausal auf die Beschwerdesymptomatik und bergen das Risiko einer Medikamentenabhängigkeit gerade bei oft chronisch verlaufenden Erkrankungen. Trotz der häufigen Schmerzsymptome sollte mit Analgetika sparsam umgegangen werden, vor allem ist auf opiatartige Substanzen zu verzichten. Ebenso fehlt für Neuroleptika (speziell zu Fluspirilen) der spezifische Indikationsbereich.

\section{Stressvermeidung}

\section{und Entspannungstherapie}

Ist Stress als möglicher Auslöser der Symptomatik identifiziert, so bietet sich zunächst an, individuelle Belastungsmomente oder Konfliktsituation zu klären und durch Verhaltensänderungen zu „entschärfen“. Eine zusätzlich Regulierung von Essensaufnahme und Schlafverhalten kann hilfreich sein. Spezifische Entspannungsverfahren wie das autogene Training, die progressive Muskelentspannung oder Yoga lassen sich in Kursen erlernen. Einschlägige Literatur mit Übungsbeispielen auf Ton und Video ist vielfältig verfügbar. 


\section{Psychotherapie}

Die Indikationsstellung und Anwendung spezifischer Psychotherapieverfahren bleibt dem ausgebildeten Facharzt, dem Psychotherapeuten und entsprechenden Fachabteilungen vorbehalten. Somatoforme Störungen sind das Ergebnis multifaktorieller, komplexer psychosozialer und intrapsychischer Enstehungsmechanismen. Diese Prozesse versucht man so weit $\mathrm{zu}$ identifizieren, dass diese in ein individuelles Therapiekonzept integriert werden können.

Psychodynamische Verfahren gehen in klassischer Weise davon aus, dass somatoforme Symptome Ausdruck einer seelischen Abwehr von unannehmbaren Wünschen oder Triebimpulsen sind. Therapeutisches Ziel hierbei ist es, tabuisierte und verdrängte Affekte und Wünsche zu erkennen und somit die Tür zu bewussten und der tatsächlichen Realität angemesseneren Bewältigungsstrategien zu öffnen. Lerntheoretische Verfahren betonen den wichtigen Einfluss von Modelllernen, sozialen Verstärkersystemen und Konditionierungsprozessen bei der Entstehung und Verfestigung der Beschwerdesymptomatik und des komplexen Krankheitsverhaltens. Diese Verfahren zielen auf eine direkte Bearbeitung dysfunktionaler Krankheitsüberzeugungen, eine Sensibilisierung gegenüber selektiver Aufmerksamkeit und Körperwahrnehmung, eine Reduktion von krankheitsbedingtem Vermeidungsoder Schonverhalten, einen Aufbau alternativer Lösungsstrategien und auf eine positive Verstärkung des gewünschten „Normalverhaltens“ hin.

\section{Wann/wie zum Psycho- therapeuten und/oder Psychiater überweisen? Der „richtige“ Zeitpunkt}

$\mathrm{Zu}$ Beginn der Therapie sollte klar ein Zeitraum definiert werden, innerhalb dessen eine oder mehrere Therapiestrategien zum Einsatz kommen. Danach sollte anhand klarer bewältigungsorientierter Zielvorgaben (z.B. „Ich möchte wieder alleine einkaufen gehen oder regelmäßig meine beste Freundin treffen “ - nicht: „Ich möchte völlig schmerzfrei sein“) beurteilt werden, ob die gewünschten Ziele in ausreichendem Maße erreicht wurden. Schon vorher sollten Vereinbarungen getroffen werden, was bei ungenügendem Therapieerfolg zu tun ist.

Ein sinnvoller Zeitraum kann drei Monate betragen. Während dieser Zeit haben ggf. Antidepressiva ausreichend Zeit, ihre Wirkung zu entfalten. Entspannungsverfahren können wirksam eingeübt und angewendet werden. Im Laufe von fünf bis sechs Gesprächen können zumeist einige aktuelle und chronische Stressoren identifiziert werden, der Patient hat Zeit genug, sich über Veränderungsmöglichkeiten Gedanken zu machen. Fatal wäre es, diese Zeit alleinig mit somatisch-diagnostischer Abklärung zu vertun - diese kann bei somatoformen Störungen keine Therapie ersetzen!

\section{Die „richtige“ Vorbereitung}

Durch die Zielvorgaben, die Wahl der Therapieverfahren, die Betonung psychosozialer und interaktioneller Aspekte wird bei unbefriedigender Therapieresponse der Weg in Richtung einer spezifischen psychiatrisch-psychotherapeutischen Behandlung gebahnt.

\section{Das eigentliche} „Motivationsgespräch“

Hierbei gilt es, den Patienten, falls nötig, zu motivieren. Das geschieht am besten, wenn der Patient merkt, dass der behandelnde Arzt den Patienten nicht „abschiebt“, sondern den bisherigen Kontakt zu ihm aufrechterhalten will. Das Verhältnis zum Arzt soll ein Vertrauensverhältnis sein, d.h. der Arzt soll den Patienten rechtzeitig auch an seinen Gedanken teilhaben lassen und ihn an Therapieentscheidungen beteiligen. Psychosoziale und psychotherapeutische Interventionen können nur mit eindeutiger Beteiligung des Patienten zum Erfolg führen. Günstig ist es, wenn der überweisende Arzt den Psychotherapeuten oder Psychiater kennt und diesen auch mit gutem Gewissen empfehlen kann. Bei ausschließlichem Einsatz psychotherapeutischer Verfahren kann die Überweisung zum psychologischen oder ärztlichen Psychotherapeuten erfolgen. Stehen zu- sätzliche differenzialdiagnostische und/oder psychopharmakologische Maßnahmen an, so sollte der Patient auf jeden Fall zum psychiatrischen Facharzt überwiesen werden. Eine wichtige Aufgabe des erstbehandelnden Arztes ist es, die Beschwerden und Funktionseinschränkungen des Patienten eindeutig als Krankheit, als behandlungsbedürftig und als behandlungswürdig einzustufen und dies dem Patienten auch bei Fehlen „harter“ somatischer Befunde $z u$ verdeutlichen und ihn dabei ernst zu nehmen. Der Wechsel von einer somatischen Diagnose zu einer psychiatrischen Diagnose kann trotz der gesellschaftlichen Vorbehalte vom Patienten vor allem dann akzeptiert werden, wenn er merkt, dass er auch ohne körperliche Erkrankung ernst genommen wird und sich durch spürbar neue Gespräche, Interventionen und ärztlich/therapeutische Kontakte tatsächlich Dinge bewegen, die für ihn von Bedeutung sind. Letztendlich leidet der Patient unter einer sehr häufigen Erkrankung, für die es mittlerweile sehr gute Therapieverfahren gibt (10).

\section{Merksätze für die Praxis}

- Die möglichst frühe Diagnose einer somatoformen Störung und die rasche Behandlung sind für den weiteren Verlauf der Erkrankung besonders wichtig

- Primäre Intervention in der haus- und allgemeinärztlichen Praxis: das empathische ärztliche Gespräch

- Schwerpunkt liegt auf der Symptombewältigung und nicht auf der körperlichen Ursachenfindung

- Aufklärung über die wahrscheinlichen psychosozialen Ursachen der Beschwerden

- angemessene organische Ausschlussdiagnostik

- Empfehlung allgemeiner ggf. individueller Maßnahmen zum Stressabbau

- Ermutigung zu körperlicher Betätigung und aktiver Lebensgestaltung trotz der Beschwerden

- Durchführung einer Psychopharmakotherapie (z.B. SSRI oder Opipramol) 
- Vermeidung von Analgetika und Benzodiazepinen

Bei unbefriedigendem Therapieverlauf sollte rechtzeitig (nach drei Monaten) eine Überweisung zu einem Psychotherapeuten/Psychiater erfolgen

\section{Summary \\ Somatoform disorders have a lifetime prevalence up to $20 \%$ and frequently a chronic course. Thus, these disor- ders represent a common challenge in medical services. The focus on so- matic complaints that are not explai- ned by somatic findings leads to a conflicting situation: Physicians keep on searching for a somatic reason for the complaints but finally both, the patient and the physician, get impati- ent and the patient himself switches (or gets switched by the physician) to another physician who is probably more specialized and who will surely "find out the reason of the distressing disorder". In the present paper we present new epidemiological data, criteria and strategies for diagnosing somatoform disorders and early in- tervention strategies. Consequently, the early and correct diagnosis and the associated therapeutic interventi- ons will reduce the distress and im- pairment of these patients and might help to avoid the development of a chronic course of the disorder.}

\section{Key Words:}

somatoform disorders - epidemio-

logy - primary care - early intervention - psychotherapy

\section{Literatur:}

1. Dilling $\mathrm{H}$, Mombour $\mathrm{W}$, Schmidt $\mathrm{MH}$ (Hrsg.). Internationale Klassifikation psychischer Störungen: ICD-10, Kapitel V (F), klinisch diagnostische Leitlinien, Weltgesundheitsorganisation. Bern; Göttingen; Toronto: Huber, 1991

2. Dilling $\mathrm{H}$, Weyerer S, Enders I. Patienten mit psychischen Störungen in der Allgemeinpraxis und ihre psychiatrische Überweisungsbedürftigkeit. In: Psychiatrische Epidemiologie. Häfner H (Hrsg.). Berlin, Heidelberg, New York, Tokyo: Springer 1978 3. Freyberger HJ, Freyberger H. Psychosomatische und verwandte Störungen. Grabe HJ, Freyberger HJ. In: Freyberger HJ, Stieglitz RD, Schneider W (Hrsg.): Kompendium Psy- chiatrie, Psychotherapie, Psychosomatische Medizin, 11., neu bearbeitete und erweiterte Auflage, orientiert an der ICD-10. Freiburg, Karger-Verlag, 2002

4. Freyberger HJ, Grabe $\mathrm{H}-\mathrm{J}$, Maier W, Janca A, Stoll KD. Opipramol (Insidon,) in der Therapie somatoformer Störungen. Fortschr Neurol Psychiat 1998; 66 (Sonderheft)

5. Grabe HJ, Freyberger HJ, Maier W, Gammel G, Stoll K-D. In der Anwendung: Opipramol bei somatoformen Störungen. Nervenheilkunde 1999; 18: 571-576

6. Grabe HJ, Freyberger HJ. Anwendungsbeobachtungen von Opipramol bei somatoformen Störungen. In: H.J. Möller und W.E. Müller (Hrsg.): Opipramol, Sigmaligand und stimmungsaufhellendes Anxioloytikum. Therapieforum Biozentrum, LinguaMed Verlags-GmbH, 2001

7. Grabe HJ, Meyer C, Hapke U, Rumpf H-J, Freyberger HJ, Dilling H, John U. The Somatoform Pain Disorder in the Community. Psychotherapy and Psychosomatics 2003; 72: 8-94

8. Grabe HJ, Meyer C, Hapke U, Rumpf H-J, Freyberger HJ, Dilling H, John U. The „Specific Somatoform Disorder" in the General Population. Psychosomatics, 2003; 44: 304-311

9. Rief W, Hiller W. Somatoforme Störungen. Bern; Göttingen; Toronto; Seattle. Hogrefe-Verlag, 1998

10. Volz HP, Möller HJ. Opipramol bei Angst- und Somatisierungsstörungen. Ergebnisse aktueller konfirmatorischer Studien. Fortschr Neurol. Psychiat 1998; 66 (Sonderheft)

11. Zintl-Wiegand A, Schmidt-Maushardt C, Leisner R, Cooper B. Psychische Erkrankungen in Mannheimer Allgemeinpraxen. In: Psychiatrische Epidemiologie. Häfner $\mathrm{H}$ (Hrsg.). Berlin, Heidelberg, New York, Tokyo: Springer 1978

\section{Korrespondenzadresse:}

PD Dr. med. Hans Jörgen Grabe

Klinik und Poliklinik für Psychiatrie und Psychotherapie der Ernst-Moritz-Arndt-Universität Greifswald im Klinikum der Hansestadt Stralsund, Rostocker Chaussee 70 18437 Stralsund

E-mail: grabeh@uni-greifswald.de
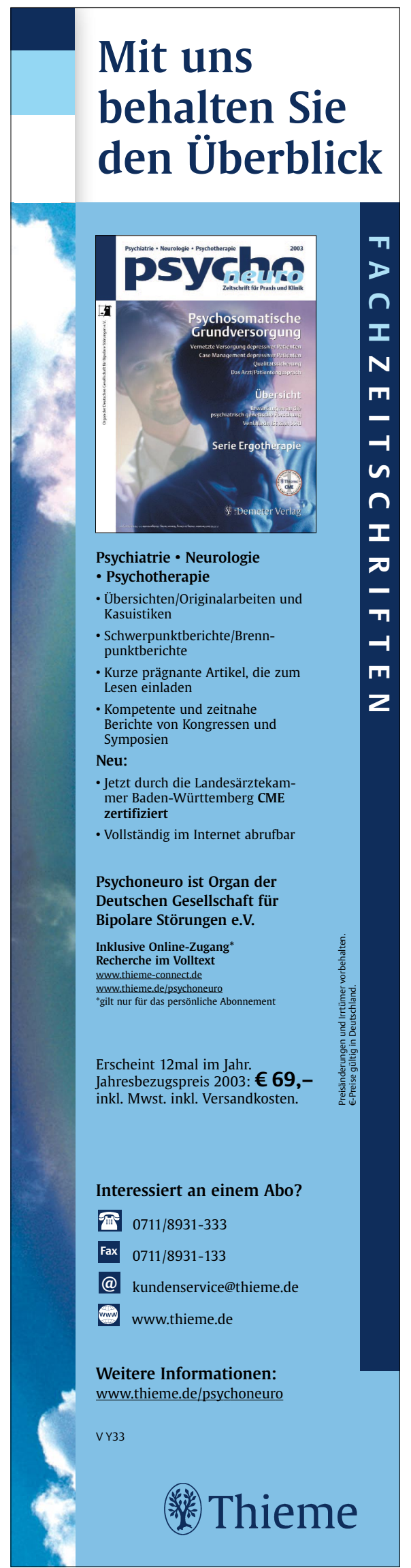